\title{
Avantages potentiels des faisceaux de mésons $\pi$ en radiothérapie $(*)$
}

\author{
J. GARSOU (**) \\ (Manuscrit reçu le 8 mai 1977)
}

\begin{abstract}
RÉSUMÉ
Après un bref rappel de l'historique de la découverte et de la production des mésons, leurs caractéristiques principales et le principe de leur production sont résumés. Les interactions avec la matière des formes positive, négative et neutre des mésons sont envisagées au niveau de la cible, du transfert du faisceau et dans les tissus biologiques soumis à celui-ci. En regard du dernier point, l'attention est principalement portée sur les caractéristiques de la dissipation de l'énergie par les particules issues des désintégrations initiées par les pions négatifs en fin de leurs parcours, caractéristiques expliquant l'intérêt de la radiothérapie par les pions négatifs.
\end{abstract}

\begin{abstract}
After a brief survey of the history of the discovery and production of mesons, their main characteristics and the principle of their production are summarized. The interactions with matter of the positive, negative and neutral forms are pointed out at the levels of target, beam transfer and biological tissues. The attention is especially drawn on the characteristics of energy deposition by particles issued from disintegrations produced by negative pions at the end of their tracks; these characteristics materialize the interest for radiotherapy by negative pions.
\end{abstract}

(*) Leçon publique destinée aux médecins candidats spécialistes en radiothérapie, exposée devant la Faculté des Sciences en dernière épreuve de l'Agrégation à l'Enseignement supérieur, le 24 septembre 1976, à Liège.

(**) Service universitaire de contrôle physique des radiations, Institut de radiologie, Hôpital de Bavière, 66, boulevard de la Constitution, 4000 Liège, Belgique. 


\section{INTRODUCTION HISTORIQUE}

En 1935, H. YukaWa émet l'hypothèse que l'agent des forces nucléaires d'échange est une particule dont la masse au repos est environ 200 fois celle de l'électron : il appelle cette particule méson parce que sa masse est intermédiaire entre celle de l'électron et celle du proton [12]. L'année suivante, C. D. Anderson et S. H. Neddermeyer découvrent le muon dans le rayonnement cosmique; le muon ne se présente que sous les formes positive et négative et n'engendre que des interactions nucléaires faibles (il est donc, actuellement, classé parmi les leptons) : ce n'est pas le méson annoncé par YUKAWA [2]. Il faut attendre les développements des émulsions nucléaires pour que, en 1947, C. F. Powell et ses collaborateurs C. M. G. Lattes, H. Muirhead et G.P. S. Occhialini découvrent le méson négatif dans le rayonnement cosmique [9] et, l'année suivante, E. GARDNER et C. M. G. LATTES produisent à Berkeley les premiers mésons artificiels [7]. En 1949, YuKaWA reçoit le Prix Nobel.

En 1961, P. H. Fowler et D. H. PERKINS annoncent les possibilités d'applications thérapeutiques des mésons négatifs [6]. Or, à ce moment, il y a à peine 6 ans que l'on exploite les bombes au ${ }^{60} \mathrm{Co}$ (leur activité maximale ne dépasse guère $2500 \mathrm{Ci}$ ) et le bétatron Siemens de $18 \mathrm{MeV}$; le bétatron Brown-Boveri Asclépitron de $35 \mathrm{MeV}$ prend son essor sur le marché ('). En 1964, H. A. ACETo dans sa thèse et en 1965, M. R. RAJu publient les premiers travaux de dosimétrie et de radiobiologie des mésons [1, 10].

En 1976, il existe cinq centres principaux de production des mésons : ce sont des installations imposantes au point qu'elles ont reçu le nom de Meson factories. Leurs caractéristiques sont résumées dans le tableau I.

TABLEAU I

Principaux CENTREs DE PRODUCTION DES MÉsONS $\pi$ (Meson factories). EN 1976

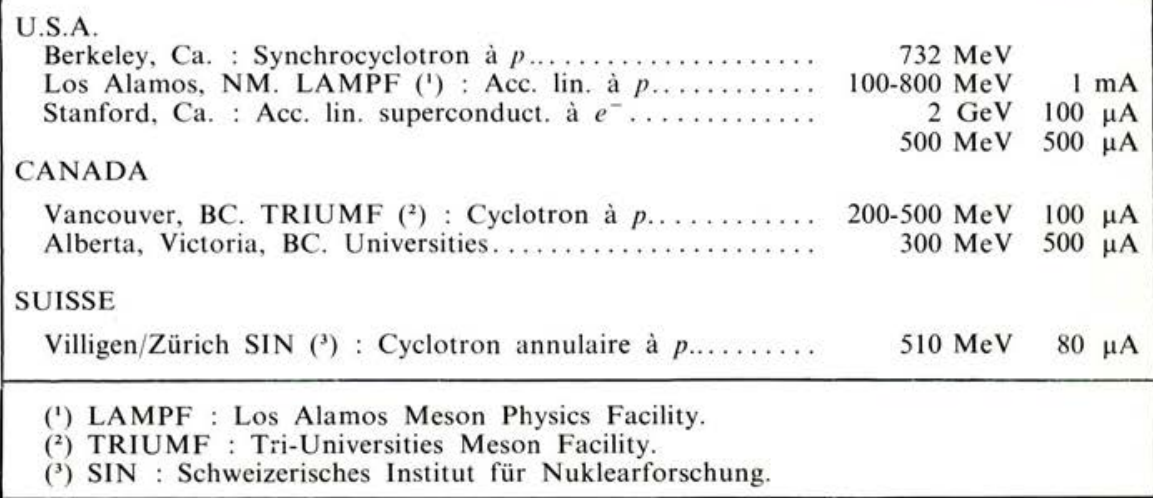

(') L'Université de Liège en acquiert un exemplaire. 
Il a été question aux États-Unis d'un projet de 30 installations de mésons. Le coût avancé pour chacune eût été de 5 millions de dollars mais il s'avère qu'en réalité ce coût est largement dépassé. Le Stanford Medical Center met, en effet, au point un générateur d'avant-garde de mésons $\pi^{-}$pour la radiothérapie qui passe pour être le moins onéreux. Si, en 1973, les espoirs étaient permis de réaliser ce générateur pour 300 millions de francs belges [8], à présent, il convient d'envisager un montant triple, l'installation complète, infrastructure comprise, revenant à 1200 millions de francs belges [4]. L'investissement est donc particulièrement lourd.

Nous essaierons, ci-après, de dégager les avantages potentiels de ces nouveaux faisceaux (potentiels, car, à ce jour, le nombre de patients traités est très faible). Pour cerner ces avantages, nous envisagerons, au préalable, les interactions de ces faisceaux avec la matière sur le plan physique et sur le plan biologique, ainsi que leur traduction dans l'application clinique. Auparavant, nous résumerons les caractéristiques principales des mésons et les modalités de leur production.

\section{I. - CARACTÉRISTIQUES PRINCIPALES}

Les mésons $\pi$ ou pions, en tant qu'agents des forces nucléaires $p-n$, se présentent sous les formes positive ou négative : leur énergie au repos $E_{0}$ est de $139,576 \pm 0,011 \mathrm{MeV}$, c'est-à-dire que leur masse au repos est 273,33 fois celle de l'électron; ils se désintègrent avec une vie moyenne de $2,55 \cdot 10^{-8} \mathrm{~s}$. L'agent des forces nucléaires $n-n$ et $p-p$ est la forme neutre $\pi^{0}$ dont l'énergie au repos est plus faible, soit $134,972 \pm 0,012 \mathrm{MeV}$; ces pions neutres se désintègrent avec une vie moyenne énormément plus courte de $0,83 \pm 0,10 \cdot 10^{-16} \mathrm{~s}$.

Les énergies au repos des pions sont donc bien intermédiaires entre celle de l'électron $(0,511 \mathrm{MeV})$ et celle du proton $(938,211 \mathrm{MeV})$.

\section{II. - PRODUCTION}

Produire des pions revient à produire des particules d'énergie au repos de $140 \mathrm{MeV}$. Cette production se réalise pratiquement par bombardement d'une cible par des particules chargées suffisamment accélérées $\left({ }^{2}\right)$, soit lourdes, tels les $p, d, \alpha$, etc., soit légères, comme les électrons qui agissent par leurs rayons $\mathrm{X}$ de freinage.

La cible est, en principe, quelconque. Sa nature chimique importe peu : pour les particules bombardantes d'énergies cinétiques élevées, les nucléons

( ${ }^{2}$ ) Par exemple, les seuils d'énergie de production des $\pi$ sont de 159,301 et $155 \mathrm{MeV}$ respectivement pour le bombardement de protons par des photons $\gamma$, de protons par des protons et des noyaux de C par des protons [10], 
peuvent être considérés comme indépendants. Les interactions intimes sont des types suivants :

$$
\begin{array}{ccc}
\begin{array}{l}
\text { particule } \\
\text { bombardante }
\end{array} & \text { nucléon } \\
p & + & p \rightarrow p+n+\pi^{+} \\
p & + & n \rightarrow p+p+\pi^{-} \\
p & + & p \rightarrow p+p+\pi^{0}
\end{array}
$$

Si l'énergie cinétique de la particule bombardante est suffisante, plusieurs pions peuvent être produits et il faut remarquer que les 3 types de pions, positif, négatif et neutre, sont formés à la fois.

La cible sera donc choisie essentiellement pour sa température de fusion élevée ainsi que pour sa bonne conductibilité thermique : c'est ainsi que, entre autres, Be est utilisé à Berkeley et à Villigen, $\mathrm{TiC}$ et $\mathrm{TiB}_{2}$ à Stanford, mais $\mathrm{C}$, $\mathrm{Cu}$ et $\mathrm{Nb}$ ont également été essayés [8].

Lors de ces bombardements, il y a production intense de neutrons de haute énergie par les réactions bien connues $(p, n)$ et $(d, n)$, ainsi que par électroet photodésintégrations.

\section{III. - INTERACTIONS AVEC LA MATIÈRE}

Sitôt produits, que deviennent les mésons $\pi$ ?

Considérons-les dans l'épaisseur de la cible, lors du transfert du faisceau, et dans leur pénétration au sein des tissus biologiques.

La cible est assez épaisse : $5 \mathrm{~cm}$ de Be à Berkeley [10], 2,54 $\mathrm{cm}$ (1 pouce) de TiC à Stanford [8], épaisseurs contrastant avec celles de $2 \mathrm{~mm}$ de W de l'accélérateur linéaire CGR MeV Neptune de 5,5 MeV et de 2,5 mm de $\mathrm{Pt}$ fritté du bétatron Brown-Boveri Asclépitron de $35 \mathrm{MeV}$, par exemple.

Le transfert du faisceau est réalisé dans des canalisations sous vide à travers un espace occupé essentiellement par un blindage imposant et un aimant de déviation. Le blindage (1,5 $\mathrm{m}$ de Fe à Stanford [8]) est une protection contre les neutrons de haute énergie dont il a été question ci-avant et contre les photons gamma de haute énergie qui seront bientôt envisagés. L'aimant de déviation permet de sélectionner l'énergie des pions $\left({ }^{3}\right)$ et le système de fentes associé, de déterminer l'intervalle d'énergie $\left({ }^{4}\right)$ de ces particules. Ce transfert du faisceau s'opère sur 5 à $10 \mathrm{~m}$ alors qu'avec les générateurs usuels de radiations, la distance source-peau est au plus de $1,10 \mathrm{~m}$.

Dans les tissus biologiques, le faisceau de mésons $\pi$ est parallèle, alors que nous sommes accoutumés à des faisceaux divergents.

La figure 1 donne un schéma de l'installation du synchrocyclotron de Berkeley où l'on remarque la longueur du transfert du faisceau. pions).

${ }^{3}$ ) Ou mieux, leur quantité de mouvement $p$ (produit de la masse et de la vitesse des

(4) Ou de quantité de mouvement, parallèlement. 


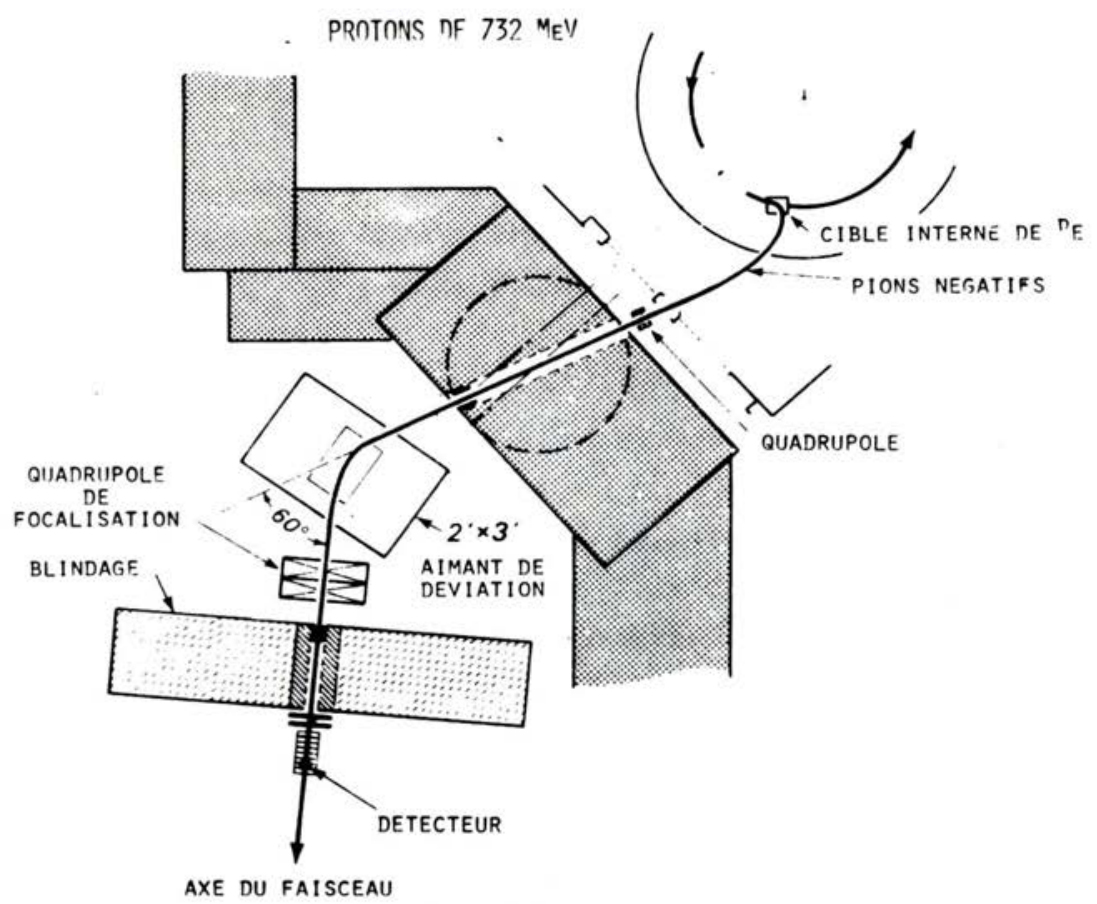

Fig. 1. - Schéma de l'installation du synchrotron de Berkeley, d'après RAJU et Richman [10].

Les pions neutres, en raison de leur fugace vie moyenne de $10^{-16} \mathrm{~s}$, se désintègrent au sein-même de la cible en 2 photons de $70 \mathrm{MeV}\left({ }^{5}\right)$, photons qui se matérialisent en paires d'électrons.

Les mésons $\pi$ positifs et négatifs peuvent être obtenus indépendamment. Le schéma de l'installation de Berkeley [10], reproduit sur la figure 1, illustre la production de pions négatifs; si l'on désire un faisceau de pions positifs, on inverse la polarité des champs magnétiques.

Nous considérerons $\pi^{+}$et $\pi^{-}$séparément.

Les mésons $\pi$ positifs, dont la vie moyenne de $10^{-8} \mathrm{~s}$ est donc de plusieurs ordres de grandeur plus élevée que celle des mésons $\pi$ neutres, se désintègrent le long du transfert du faisceau en muons positifs, $\mu^{+}$, et neutrinos, $v_{\mu}$, associés à l'apparition du muon.

Les muons positifs ont une énergie au repos de 105,6 MeV; leur énergie cinétique est ici de $4,12 \mathrm{MeV}$; ils se désintègrent avec une vie moyenne de $2,2 \cdot 10^{-6} \mathrm{~s}$ en un électron positif $e^{+}$, un neutrino associé à l'électron et un antineutrino associé à la disparition du muon. La distribution des énergies cinétiques de ces électrons est analogue à celle d'un spectre continu $\beta$ : l'énergie

$\left({ }^{5}\right)$ Lorsque les pions neutres sont au repos. 
maximale est de $54 \mathrm{MeV}$, l'énergie au pic est de $30 \mathrm{MeV}$. Le flux de pions positifs diminue avec la distance du transfert : en moyenne, il n'est plus que de $50 \mathrm{p}$. cent après $5 \mathrm{~m}$, de $25 \mathrm{p}$. cent après $10 \mathrm{~m}$.

La figure 2 montre, dans une émulsion photographique, 4 traces de pions suivies chacune après désintégration d'une trace de muon, elle-même suivie après désintégration d'une trace d'électron: la densité d'ionisation, illustrée par la densité linéaire de grains développés diminue du pion au muon et à ¡électron.

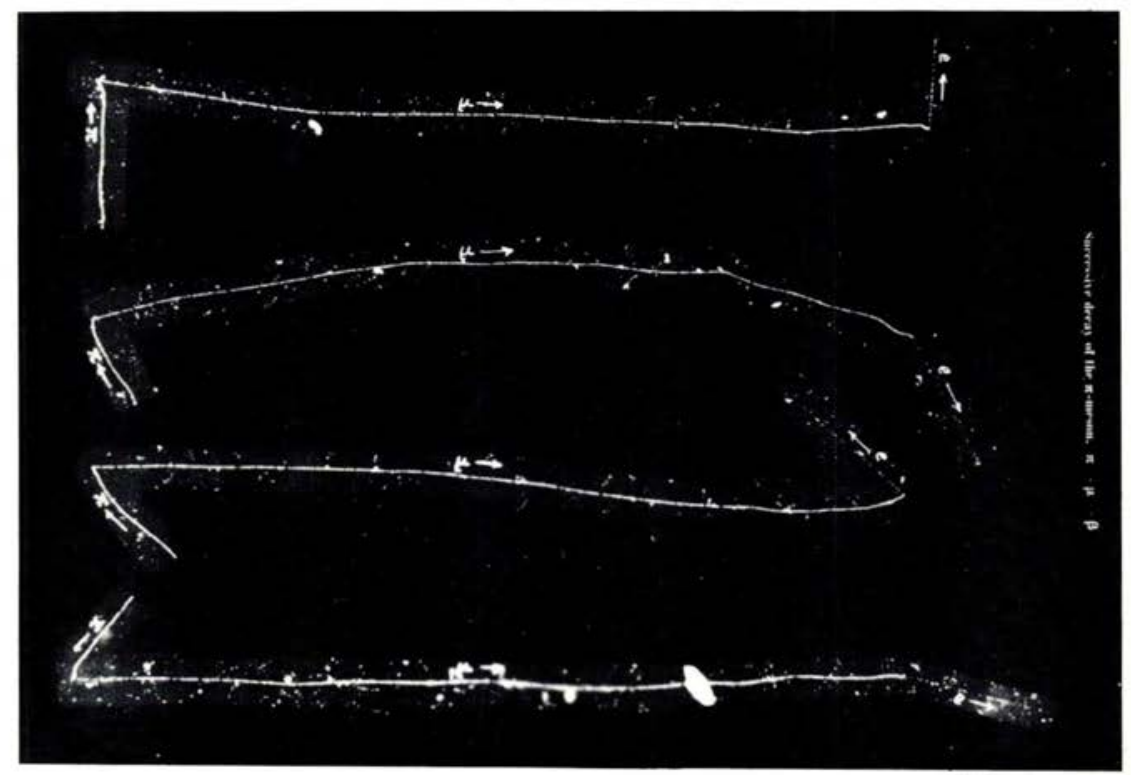

Fig. 2. - Traces successives de pion. muon et électron dans une émulsion nucléaire.

Dans les tissus biologiques, les pions, tout comme les muons et les électrons, dissipent leur énergie par excitations et collisions ionisantes; en fin de parcours, la répulsion coulombienne les empêche de réagir avec les noyaux : il y a achèvement des désintégrations.

Les mésons $\pi$ négatifs, au long du transfert du faisceau et dans les premiers centimètres de pénétration dans les tissus, se comportent exactement comme les mésons $\pi$ positifs : ils se désintègrent en muons négatifs et ceux-ci en électrons négatifs, les neutrinos associés étant identiques. Mais en fin de parcours, le pion négatif est capté par une orbitale atomique externe d'où il tombe en cascade sur des orbitales de plus en plus profondes. Il y a émission concomittante de rayons $\mathrm{X}$ caractéristiques mésiques, analogues aux rayons $\mathrm{X}$ caractéristiques électroniques, mais d'énergie plus élevée : par exemple, dans le cas de l'oxygène, la transition mésique $2 p-1 s$ est de $160 \mathrm{keV}$ tandis que les photons $\mathrm{K}_{\text {, }}$ ont moins de $0.5 \mathrm{keV}$. 
Or, le rayon des orbites mésiques est la $273^{\mathrm{e}}$ partie du rayon des orbites électroniques. Le temps de passage du méson $\pi^{-}$dans le noyau est important : le méson est finalement capté par ce noyau. Un noyau mésique est ainsi formé : il éclate aussitôt en diverses particules qui s'éloignent les unes des autres comme sur les branches d'une étoile.

Sur la figure 3 , un pion négatif dont la trace dans l'émulsion nucléaire est issue d'une étoile de production, provoque l'éclatement d'un noyau : du site de celui-ci partent les traces de particules lourdes chargées à la fois plus courtes et plus denses.

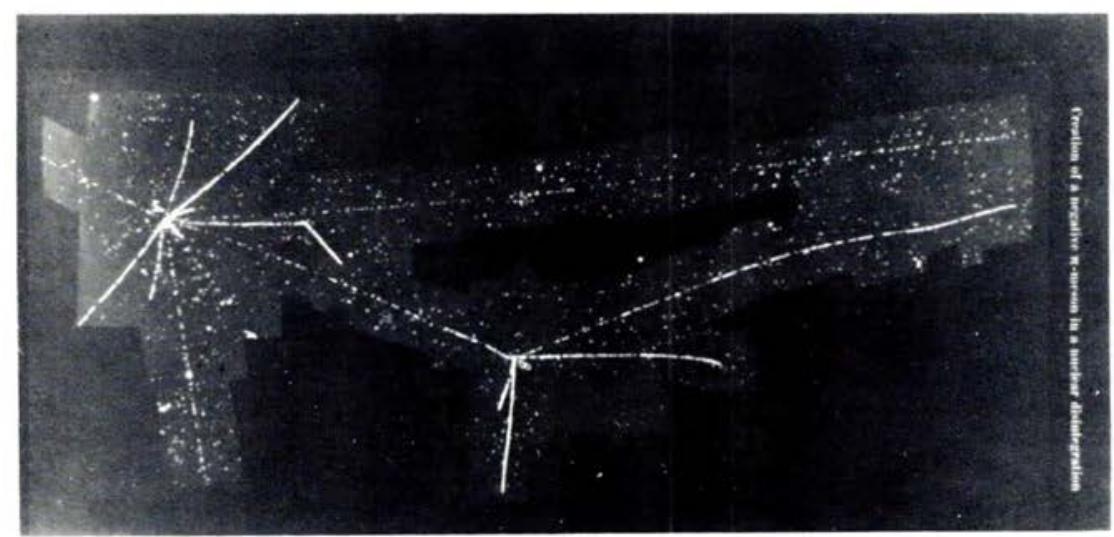

FIG. 3. - Trace dans une émulsion nucléaire d'un pion négatif issu de l'étoile A de production et entrainant en B la désintégration d'un noyau dont les traces des particules chargées résultantes sont reconnaissables par une densité linéique de grains plus élevée.

La section efficace de capture des pions négatifs augmente avec le nombre atomique $Z$ des noyaux, mais cette progression ne se remarque guère dans l'intervalle restreint des $\mathrm{Z}$ des principaux atomes constitutifs des tissus mous, c'est-à-dire ${ }_{6} \mathrm{C},{ }_{7} \mathrm{~N}$ et ${ }_{8} \mathrm{O}$ à raison de 11,$3 ; 2,8$ et 75 p. cent respectivement. $\mathrm{Si}$ les noyaux de carbone captent 20 p. cent des pions négatifs, les noyaux d'azote, 3 p. cent et les noyaux d'oxygène, $73 \mathrm{p}$. cent, les noyaux de $\mathrm{Z}$ supérieur à 8 sont responsables de $4 \mathrm{p}$. cent des captures : on voit l'importance de l'oxygène dans la capture des pions négatifs.

Lors d'une capture nucléaire d'un pion négatif, les $140 \mathrm{MeV}$ de l'énergie au repos du pion se répartissent comme suit : $40 \mathrm{MeV}$ sont absorbés par l'excitation du noyau et la rupture des liaisons nucléaires, $100 \mathrm{MeV}$ se distribuent en énergie cinétique d'une particule de charge unitaire d'énergie cinétique de l'ordre de $15 \mathrm{MeV}$ (dans 1 cas sur 2, il s'agit d'un proton; dans 1 cas sur 3 , d'un deuton; dans 1 cas sur 6 , d'un triton), d'une particule $\alpha$ d'énergie cinétique de $8 \mathrm{MeV}$ environ, d'un noyau de $\mathrm{Z}>3$ d'énergie cinétique de $4 \mathrm{MeV}$ et de 3 neutrons d'énergie cinétique globale de $73 \mathrm{MeV}$. Donc, en moyenne, 6 particules sont mises en mouvement par capture de pion, mais il s'y ajoute 2 p. cent de photons gamma d'énergie maximale de $100 \mathrm{MeV}$ : tel est le bilan d'une capture de pion négatif par un noyau d'oxygène, le cas le plus fréquent. 
Si les particules de charge unitaire et les $\alpha$ ont des parcours dans les tissus inférieurs à $1 \mathrm{~cm}$, par contre les protons mis en mouvement par les neutrons ont des parcours dépassant nettement le centimètre.

La globalisation de ces événements élémentaires se traduit par une courbe de dose absorbée en profondeur. La courbe représentée sur la figure 4 se rapporte à un faisceau réel de pions négatifs c'est-à-dire contaminé par $65 \mathrm{p}$. cent de pions, $25 \mathrm{p}$. cent d'électrons et $10 \mathrm{p}$. cent de muons.

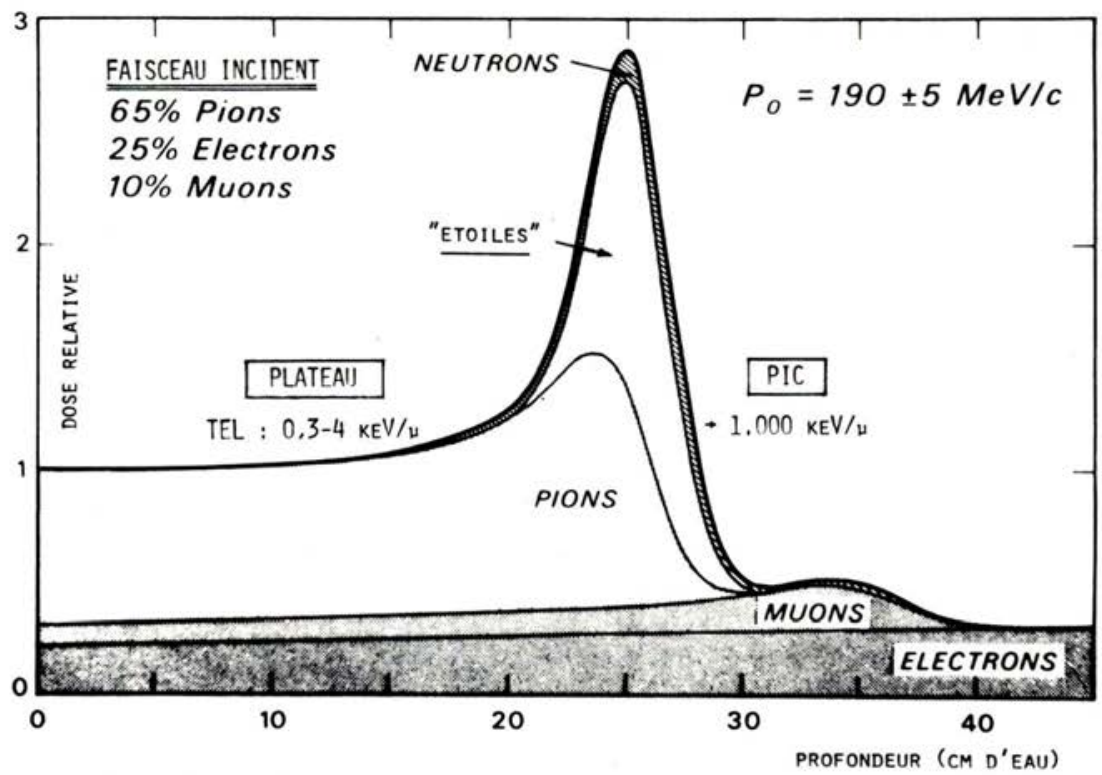

FIG. 4. - Variations en fonction de la profondeur dans l'eau des contributions à la dose absorbée des électrons, muons, pions, particules lourdes chargées et neutrons pour un faisceau réel de pions négatifs, d’après RAJU et RichmaN [10].

La courbe mesurée est la somme de 4 contributions à la dose absorbée :

1) celle des électrons provenant de la désintégration des muons et de la matérialisation des photons de $70 \mathrm{MeV}$ issus de la désintégration des pions neutres:

2) celle des muons négatifs, marquée par un léger pic de Bragg, le parcours de ces muons étant de $30 \mathrm{p}$. cent supérieur à celui des pions;

3) celle des pions négatifs avec un pic de Bragg plus accusé;

4) celle des particules lourdes chargées des étoiles de désintégration, qu'englobe la contribution relative aux protons mis en mouvement par les neutrons.

Seuls, les pions négatifs présentent cet accroissement de la dose absorbée au niveau du pic de Bragg; avec les pions positifs, la courbe de dose absorbée en profondeur résulte uniquement de la somme des trois premières contributions signalées ci-avant. 
Ces courbes de dose absorbée en profondeur des pions négatifs présentent essentiellement 2 parties :

1) un plateau le long duquel, depuis la surface, la dose absorbée ne varie guère, dose absorbée due à la dissipation de l'énergie de pions, de muons et d'électrons négatifs avec des transferts d'énergie linéiques (TEL) de 0.3 à $4 \mathrm{keV} / \mu \mathrm{m}$ :

2) un pic où la dose absorbée est subitement multipliée par 2, 3 jusque par 6, dose absorbée due à la dissipation de l'énergie de pions, de muons et d'électrons comme le long du plateau, mais, en plus, à la dissipation de l'énergie des $p, d, t, \alpha$, noyaux de $\mathrm{Z}>3, p$ mis en mouvement par les $n$, avec des TEL s'étalant jusqu'à $1000 \mathrm{keV} / \mu \mathrm{m}$.

C'est sur cette différence de TEL aux niveaux du plateau et du pic que se fonde l'intérêt porté aux pions négatifs.

Le TEL conditionne en effet :

1) l'efficacité biologique relative (EBR);

2) la possibilité de récupération des tissus irradiés en fonction du fractionnement de la dose administrée;

3) l'effet oxygène.

1) L'EBR augmente avec le TEL, jusqu'à ce que l'énergie déposée devienne supérieure à l'énergie nécessaire pour un effet cellulaire létal; l'EBR diminue alors avec le gaspillage de l'énergie. Cette variation de l'EBR avec le TEL est illustrée par la courbe de G. W. BARENDSEN [5] reprise à la figure 5 .

2) Quant à la possibilité de récupération des tissus irradiés lorsque la dose administrée est fractionnée dans le temps, si elle se manifeste avec les radiations de TEL faible (rayons X et $\gamma$, électrons, $\mu^{+e t^{-}}$et $\pi^{+\mathrm{et}^{-}}$le long du plateau de la courbe de dose en profondeur), elle disparaît avec les radiations de TEL élevé, telles les particules $\alpha$.

3) L'effet oxygène est quantifié par le facteur d'amplification de l'effet biologique par l'oxygène (l'OER, oxygen enhancement ratio, des Anglo-Saxons). facteur par lequel un effet biologique donné sur des cellules anoxiques est multiplié lorsque l'oxygène vient à être présent dans ces cellules $\left(^{6}\right)$.

La variation de ce facteur en fonction du TEL est illustrée sur le diagramme supérieur de la figure 5 [5]; après avoir présenté une valeur allant de 2,7 à 2,5 (admettons 3) pour des TEL croissant jusqu'à $10 \mathrm{keV} / \mu \mathrm{m}$, ce facteur tombe progressivement à une valeur unitaire pour un TEL de l'ordre de $160 \mathrm{keV} / \mu \mathrm{m}$.

Donc, en ce qui regarde la dissipation de l'énergie des pions négatifs au niveau du plateau de la courbe de dose en profondeur (c'est aussi le cas des rayons $\mathrm{X}$, gamma, électrons que nous connaissons) :

1) l'EBR est le plus faible : de l'ordre de 1;

$\left({ }^{\circ}\right)$ Nous prendrons l'abréviation FABO. 
2) les tissus récupèrent entre les séances d'irradiation;

3) le Facteur d'Amplication de l'Effet Biologique par $\mathrm{O}_{2}$ est le plus élevé : de l'ordre de 3.

Si nous envisageons la dissipation de l'énergie au niveau du pic de dose absorbée de la courbe de dose en profondeur, le spectre de TEL et ses diverses composantes sont donnés dans le diagramme inférieur de la figure 5 : successivement des faibles TEL vers les TEL élevés, les composantes des électrons, muons, pions, protons (mis en mouvement par les neutrons de haute énergie puis les protons issus des étoiles de désintégration), particules alpha et noyaux de $\mathrm{Z} \geqslant 3$ (exemple : ${ }^{6} \mathrm{Li}$ et ${ }^{12} \mathrm{C}$ ) issus également des étoiles de désintégration.

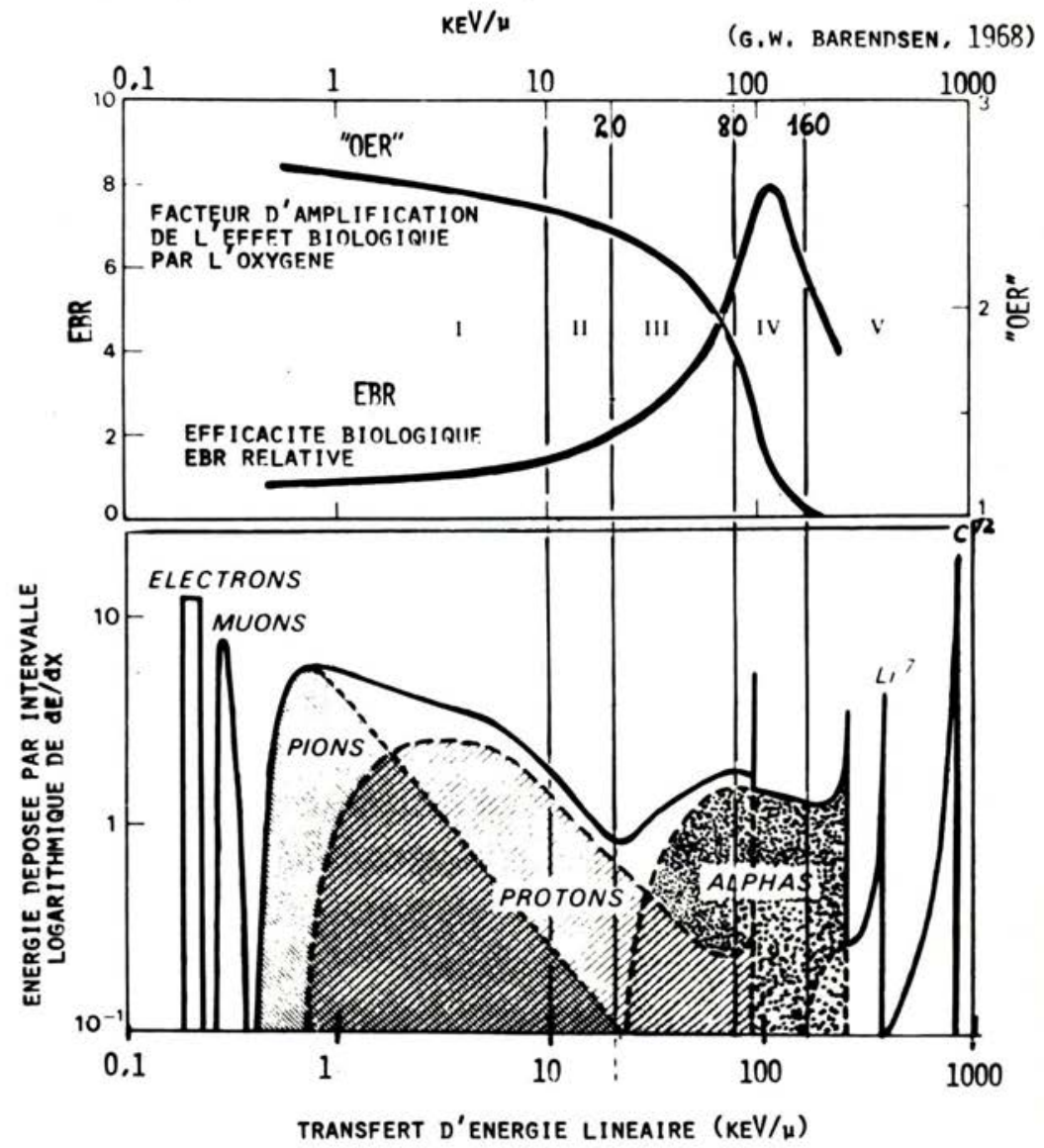

FIG. 5. - En haut : Variation en fonction du transfert d'énergie linéique du facteur d'amplification de l'effet biologique par l'oxygène et de l'efficacité biologique relative, d'après BARENDSEN [5], En bas: Spectre de TEL de la dissipation d'énergie au niveau du pic de dose absorbée en profondeur dans le cas d'un faisceau réel de mésons $\pi$ négatifs, d'après Raju et Richman [10]. 
Dans une large approximation, malgré les différences, on assimile ce spectre de TEL à celui des neutrons de $15 \mathrm{MeV}$ dans les tissus.

Par conséquent, au niveau de ce pic de la courbe de dose en profondeur, l'EBR et le FABO prennent des valeurs intermédiaires entre celles correspondant à des TEL respectivement peu et très élevés : on applique en moyenne la valeur de 2-2,5 à l'EBR et celle de 1,6 au FABO. Les tissus biologiques irradiés par des doses fractionnées dans le temps présentent plus de récupération.

Mais, attardons-nous un instant à l'effet oxygène. Les tumeurs comportent, en périphérie, des cellules bien oxygénées, tandis qu'au centre, en fonction de maints facteurs, dont le volume tumoral, une certaine quantité de cellules sont hypoxiques. D'une part, au niveau du plateau de la courbe de dose en profondeur des pions négatifs (comme le long des trajectoires des rayons $\mathrm{X}$ et $\gamma$ des électrons), l'effet biologique sur les cellules oxygénées est le triple de l'effet biologique sur les cellules hypoxiques et inversement. D'autre part, au niveau du pic de cette courbe de dose, l'effet biologique sur les cellules oxygénées est 1,6 fois aussi élevé que l'effet biologique sur les cellules anoxiques et inversement. Ceci revient à dire que, si nous faisons coïncider le pic de dose absorbée et le centre de la tumeur, les cellules hypoxiques y subissent un effet pratiquement double de celui subi par des radiations de faible TEL. Les tissus biologiques sains oxygénés susjacents sont atteints par des trajectoires à TEL faible et à FABO plus élevé, sans aucun doute, mais à EBR et débit de dose absorbée nettement plus faibles.

\section{Problème PRATIQúe de L'IRRADIATION D'UNE TUMEUR}

Sous la peau, cette tumeur se trouve à une certaine profondeur; elle présente, selon l'axe central du faisceau, une épaisseur donnée, et, perpendiculairement à cet axe, une largeur déterminée.

Les questions suivantes se posent :

1) Quelle énergie de pions négatifs faut-il produire pour que le pic de la courbe de dose en profondeur corresponde à la profondeur du centre de la tumeur?

2) Comment ajuster la largeur du pic à la largeur de la tumeur ?

3) Comment se comporte latéralement en profondeur un faisceau de pions négatifs ?

1) La figure 6 donne la relation graphique entre l'énergie des pions et leurs parcours dans l'eau. Pour des tumeurs situées entre 6 et $16 \mathrm{~cm}$ de profondeur, il faut recourir à des pions négatifs de 40 à $70 \mathrm{MeV}$.

2) Les courbes de dose en profondeur de la figure 7 se rapportent à des faisceaux définis par des fentes de largeur croissante (il s'agit du système de fentes, déjà vu, associé à l'aimant de déviation, sélecteur de l'énergie et définissant l'intervalle d'énergie ou de quantité de mouvement). Plus la fente est large, plus le pic s'élargit, mais au détriment de sa hauteur.

3) Les courbes isodoses dans l'eau de la figure 8 se rapportent à un faisceau réel d'un diamètre de $10 \mathrm{~cm}$ en surface de l'eau. Ces courbes isodoses s'évasent 


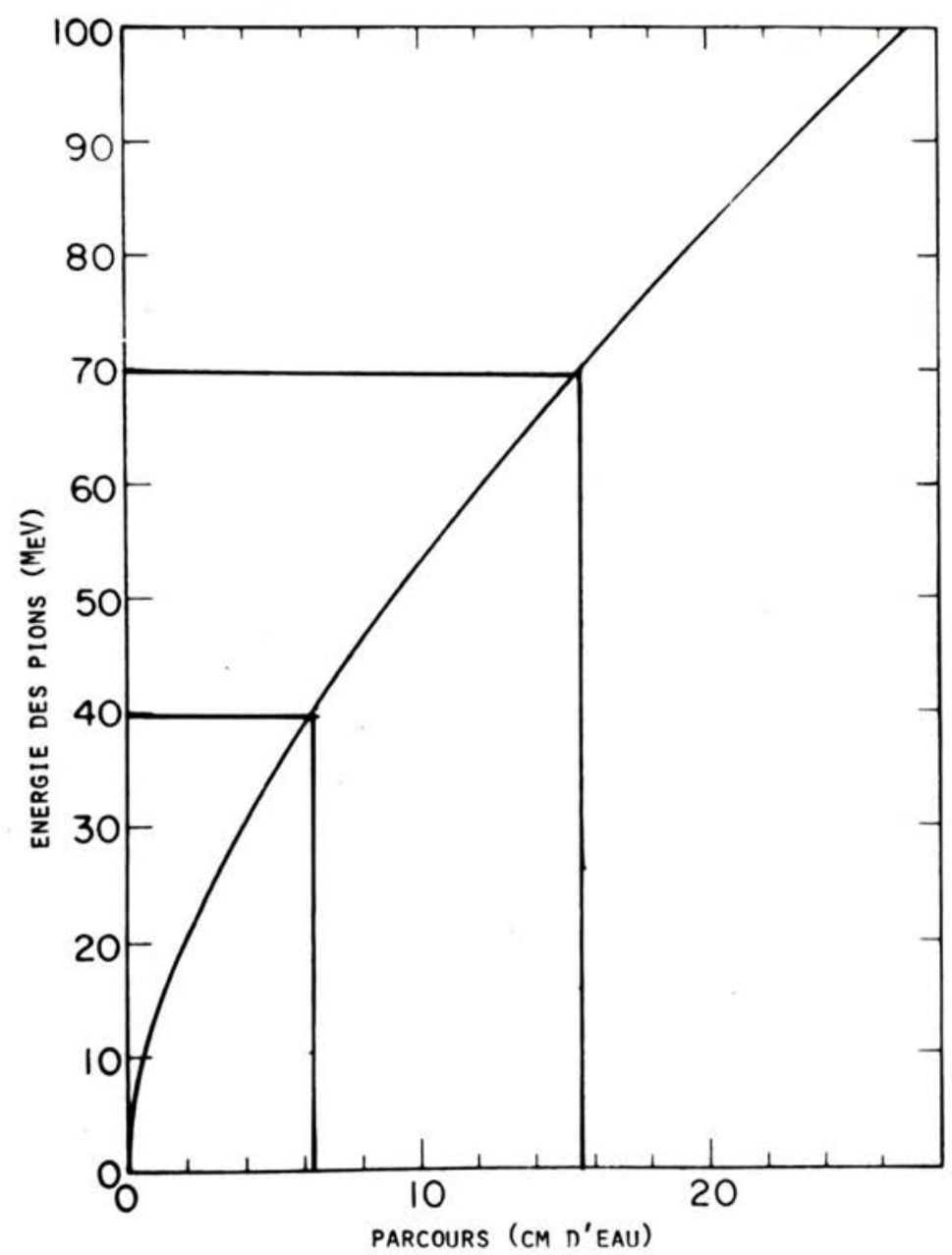

FIG. 6. - Relation graphique entre le parcours des pions négatifs exprimés en centimètres d'eau et l'énergie des pions en MeV, d'après Raju et Richman [10], montrant que les énergies pratiquement utilisables des pions s'étendent de 40 à $70 \mathrm{MeV}$.

quelque peu dans l'eau tout comme celles d'un faisceau d'électrons accélérés par un bétatron; leur forme dans un autre plan contenant l'axe central du faisceau ne s'obtient pas a priori par symétrie de révolution des premières autour de l'axe du faisceau, comme cela s'observe aussi avec un faisceau d'électrons de bétatron.

Dans le cas de la figure 8 , la courbe isodose de 80 p. cent peut circonscrire une tumeur épaisse de $2 \mathrm{~cm}$. 


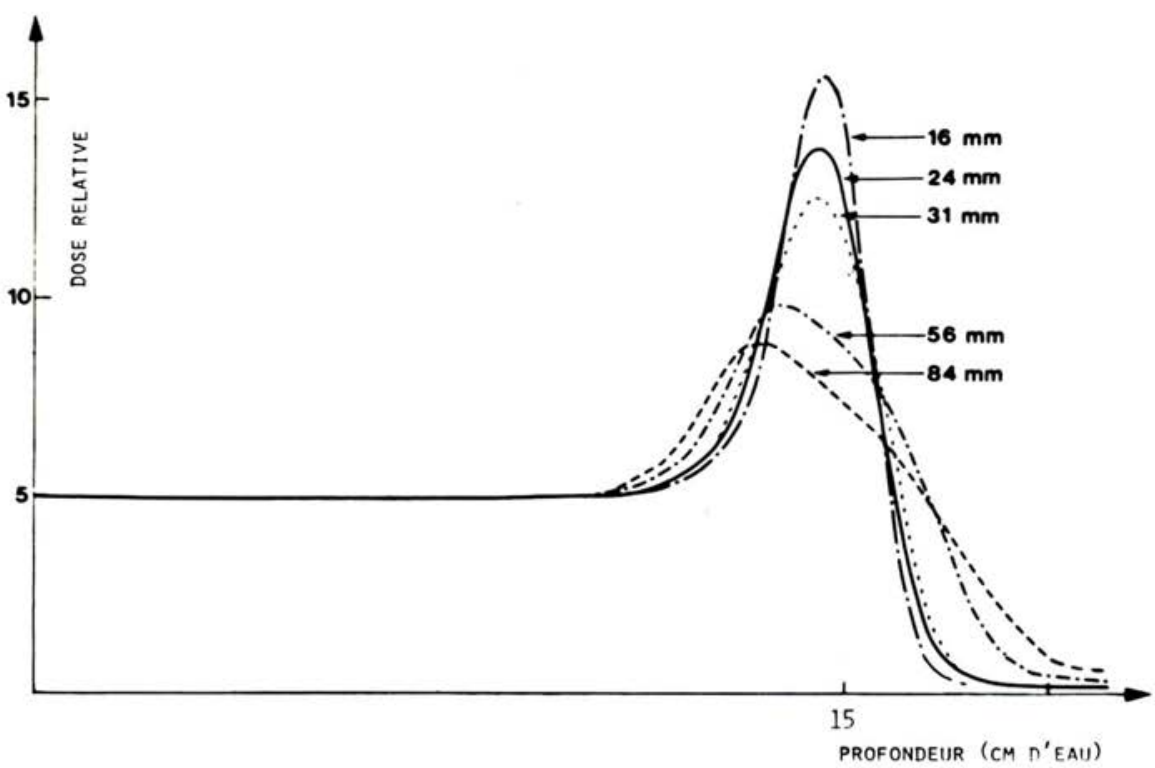

Fig. 7. - Courbe de dose absorbée en profondeur montrant l'étalement et l'aplatissement du pic à mesure qu'augmente la largeur des fentes à la sortie de l'analyseur magnétique, d'après BAARLI et coll. [3].

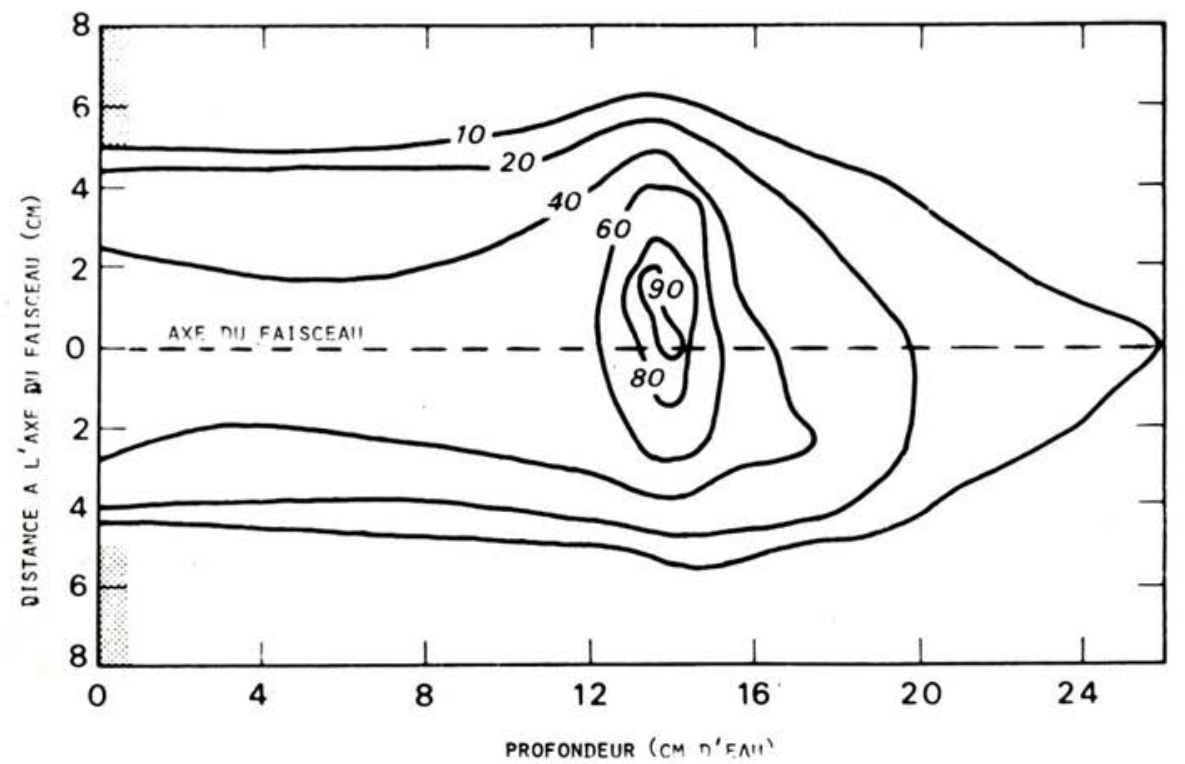

FIG. 8. - Courbes isodoses dans l'eau d'un faisceau réel de pions négatifs, d'après RAJU et RICHMAN [10]. 


\section{IV. - AVANTAGES POTENTIELS}

Rassemblons les divers points que nous venons d'exposer.

\section{Distribution de LA DOSE abSORbÉE EN PROFONDEUR}

Nous avons porté sur la figure 9 les courbes de dose absorbée en profondeur relatives à des rayons $\gamma$ du ${ }^{60} \mathrm{Co}$ et à des mésons $\pi$ négatifs; ces courbes sont normalisées à $100 \mathrm{p}$. cent au niveau de la tumeur que nous situons entre 13 et $17 \mathrm{~cm}$ de profondeur.
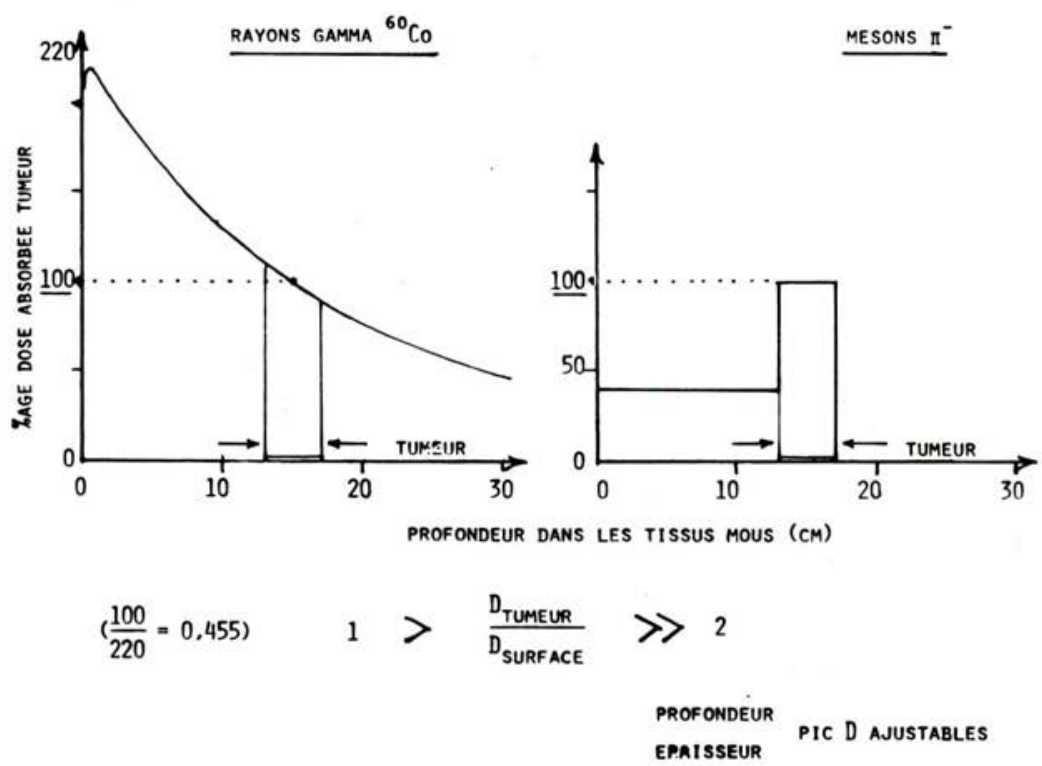

Fig. 9. - Variation de la dose absorbée en fonction de la profondeur dans l'eau dans le cas d'un faisceau de rayons $\gamma$ du ${ }^{60} \mathrm{Co}$ et d'un faisceau de mésons $\pi$ négatifs, la courbe en cause étant normalisée à $100 \mathrm{p}$. cent au niveau moyen d'une tumeur s'étendant de 13 à $17 \mathrm{~cm}$ de profondeur, d'après Raju et Richman [10].

Avec les rayons $\gamma \mathrm{du}{ }^{60} \mathrm{Co}$, la dose à la tumeur est nettement inférieure à la dose en surface $\left(^{7}\right)$, (dans ce cas, $100 / 220=45,5$ p. cent) et la dose intégrale absorbée par tout l'organisme $\left({ }^{8}\right)$ est très élevée. Pour administrer à la tumeur une dose absorbée plus élevée, il faut viser cette tumeur avec deux ou plusieurs faisceaux d'angles d'incidence différents; mais dans ce cas, la dose intégrale absorbée est encore accrue.

(7) Exactement à 4 ou $5 \mathrm{~mm}$ sous la surface, selon le champ d'irradiation.

( $\left.{ }^{8}\right)$ Évaluée par la surface délimitée sous la courbe de dose absorbée en profondeur. 
Avec les mésons $\pi^{-}$, par contre, nous avons vu que la dose à la tumeur (c'est-à-dire au pic de la courbe de dose) est nettement plus élevée que la dose à la surface ou le long du plateau de la courbe de dose, et la dose intégrale absorbée est nettement plus faible que dans le cas précédent du faisceau de rayons $\gamma \mathrm{du}{ }^{60} \mathrm{Co}$. Un seul faisceau peut suffire pour délivrer à la tumeur une dose plus élevée qu'aux tissus sains voisins; de plus, profondeur et largeur du pic de dose absorbée peuvent être ajustées à la profondeur et à l'épaisseur de la tumeur.

\section{Distribution DE L'ÉQUIVALENT DE DOSE EN PROFONDEUR}

L'équivalent de dose est le produit « dose absorbée $\times$ EBR ».

Avec les rayons $\gamma$ du ${ }^{60} \mathrm{Co}$ ( fig. 10 ), prenons $\mathrm{EBR}=1$ pour la simplicité de l'exposé et cette EBR est constante tout au long du trajet du faisceau. Comme pour la dose absorbée, l'équivalent de dose à la tumeur est nettement inférieur à l'équivalent de dose en surface, soit ici 45,5 p. cent.

Avec les mésons $\pi^{-}$, l'EBR est aussi égale à 1 le long du plateau de la courbe de dose, tandis qu'elle s'élève à 2-2,5 au niveau de son pic; par conséquent,

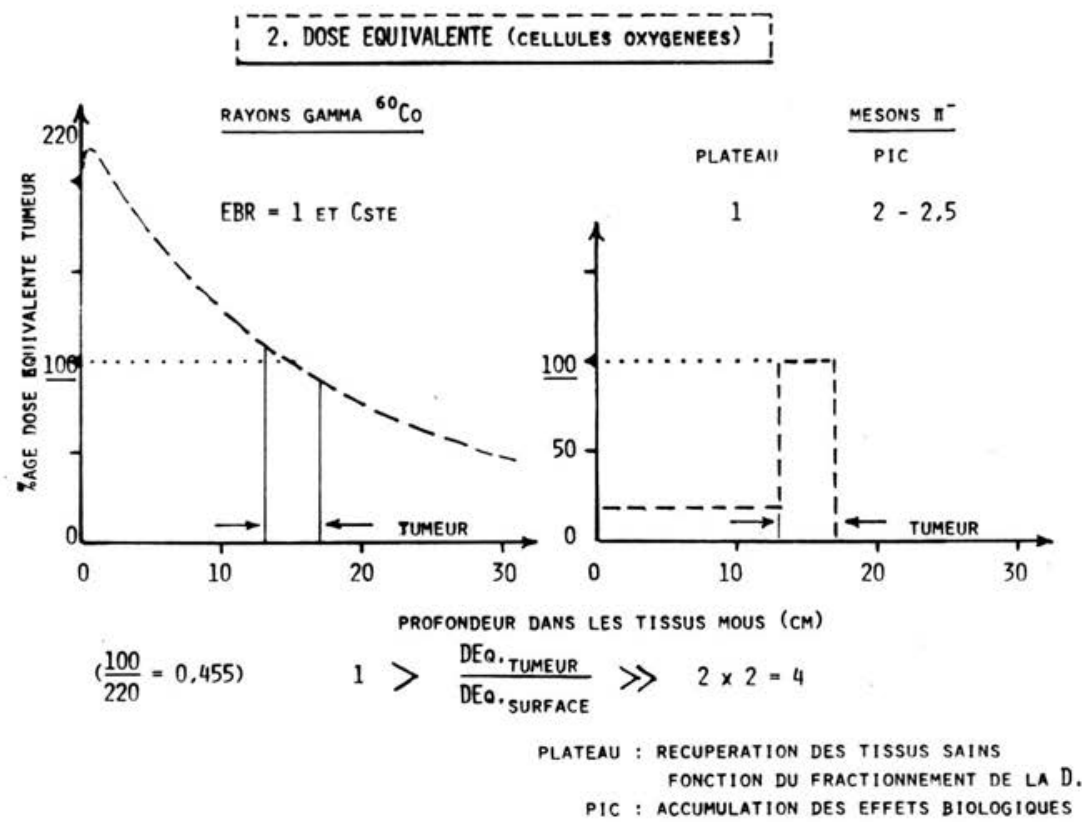

FIG. 10. - Variation de l'équivalent de dose (produit de la dose absorbée par le coefficient d'efficacité biologique relative) relatif aux cellules oxygénées en fonction de la profondeur dans l'eau pour un faisceau de rayons $\gamma \mathrm{du}{ }^{60} \mathrm{Co}$ et pour un faisceau de mésons $\pi$ négatifs, la courbe étant normalisée à $100 \mathrm{p}$. cent au niveau de la tumeur profonde, d'après RAJU et Richman [10]. 
l'équivalent de dose à la tumeur est, au moins, nettement plus élevé que $2 \times 2=4$ fois $\left({ }^{9}\right)$ l'équivalent de dose en surface.

Mais, de plus, au niveau du plateau de la courbe de dose, les tissus sains peuvent récupérer en fonction du fractionnement de la dose, tandis qu'au pic, il y a accumulation des effets biologiques, quel que soit le mode d'administration de la dose.

3. En ce qui concerne l'effet biologique sur les cellules anoxiques de la tumeur ( fig. 11), cellules les plus difficilement atteintes par la radiothérapie actuelle, nous avons vu qu'avec les rayons $\gamma$ du ${ }^{60} \mathrm{Co}$, le FABO est de l'ordre de 3 et que l'effet biologique sur les cellules anoxiques est, par conséquent, $33 \mathrm{p}$. cent de l'effet biologique sur les cellules oxygénées. Il s'ensuit que l'équivalent de dose pour les cellules anoxiques est $33 / 220=15$ p. cent de l'équivalent de dose pour les cellules oxygénées de la surface $\left({ }^{10}\right)$.

\section{DOSE EQUIVALENTE (CELL. HYPOXIQUES DE LA TUMEUR)}

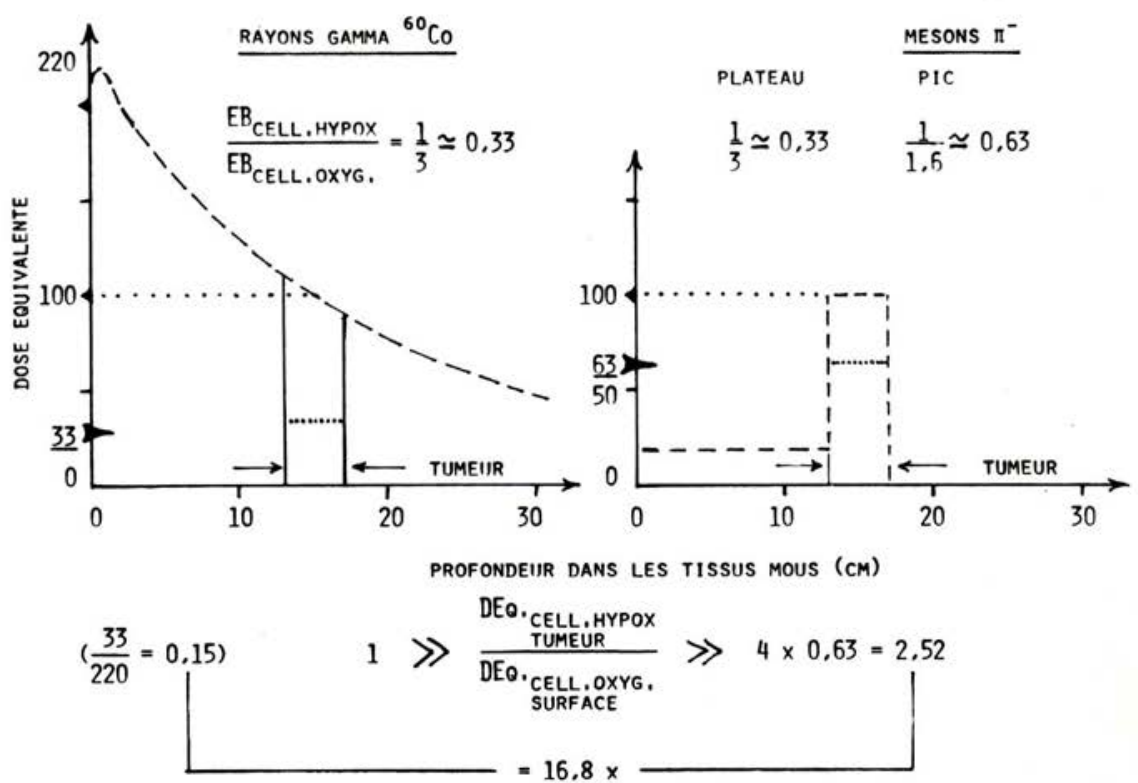

FIG. 11. - Introduction sur les diagrammes de la figure 10 des différences d'effet biologique sur les cellules hypoxiques de la tumeur et sur les cellules oxygénées, d'après RAJU et Richman [10].

$\left({ }^{9}\right)$ Nous avons pris, dans ce produit, les valeurs les plus faibles de chacun des facteurs.

$\left({ }^{10}\right)$ Même remarque que précédemment : exactement à 4 ou $5 \mathrm{~mm}$ sous la surface. 
Avec les mésons $\pi^{-}$, ce FABO décroît de 3 au niveau du plateau à 1,6 au niveau du pic et l'équivalent de dose pour les cellules hypoxiques de la tumeur reste nettement supérieur à $4 \times 0,63$ (l'inverse de 1,6 ), soit 2,52 fois l'équivalent de dose pour les cellules oxygénées en surface ou le long du plateau, soit environ 17 fois plus que dans le cas du ${ }^{60} \mathrm{Co}$.

En résumé, les trois avantages qui se multiplient, au contraire de ce qui s'observe avec les gamma du ${ }^{60} \mathrm{Co}$, découlent essentiellement du fait que la capture des pions négatifs par les noyaux met en mouvement des particules lourdes chargées à TEL relativement élevé.

Ces avantages bien précisés par des études radiobiologiques doivent, à présent, être concrétisés par des essais cliniques développés.

Après avoir précisé les caractéristiques principales des mésons $\pi$ et les éléments de leur production, nous avons vu, à la lumière de leurs interactions avec la matière, que c'étaient les mésons $\pi$ négatifs qui étaient particulièrement intéressants en radiothérapie. Les mesons $\pi$ négatifs sont susceptibles de pallier les insuffisances des faisceaux de radiations actuellement utilisés, mais au prix d'un énorme investissement.

\section{REMERCIEMENTS}

Notre reconnaissance est entière aux Professeurs J. Duchesne, L. Winand et $\mathrm{J}$. Closon pour la documentation qu'ils mirent à notre disposition, aux Professeurs D. Blanc et B. Grinberg pour avoir accepté d'être à la fois nos juges et conseillers, aux Professeurs et Docteurs L. H. Lanzl, C. Richman, J. F. Dicello, M. A. Bagshaw, J. Dutreix, J. Baarli, R. Loevinger et R. Madey pour les discussions intéressantes et bienveillantes qu'ils acceptèrent d'avoir avec nous et pour la documentation qu'ils voulurent bien nous confier.

\section{BIBLIOGRAPHIE}

[1] ACEto H. A. A feasibility study of the therapeutic possibilities of pi-mesons, Ph. D. Thesis, Lawrence Radiation Laboratory Report UCRL-11482, 1964.

[2] Anderson C. D.. Neddermeyer S. H. Phys. Rev.., 1936. 50, 263: 1937. 51, 884.

[3] Baarli J., Nordell B., Sullivan A. H. Setting up of the $\pi$ E3 negative pion beam at SIN for radiobiological irradiations. Atomkern., 1976, 27, 175-176.

[4] Bagshaw M. A. Communication privée, 1976.

[5] Barendisen G. W. Responses of cultured cells, tumours and normal tissues to radiations of different linear energy transfer. In: Current topics in radiation research (M. Ebert et A. Howard. Eds), Amsterdam. North Holland Publishing Co.. 1968, 4, 293-356.

[6] Fowler P. H., Perkins D. H. The possibility of therapeutic applications of beams of negative $\pi$-mesons. Nature (London), 1961, 189, 524-528.

[7] Gardner E.. Lattes C. M. G. Science, 1948, 107, 270.

VOL. $12-\mathrm{N}^{\circ} 3$ 
[8] Kaplan H. S., Schwettman H. A., Fairbank W. M., Boyd D., Bagshaw M. A. A hospital based superconducting accelerator facility for negative pi-meson beam radiotherapy. Radiol., 1973, 108, 159-172.

[9] Powell C. F., Lattes C. M. G., Muirhead H., Occhialini G. P. S. Nature, 1947, 160, 453.

[10] RaJU M. R., Richman C. Negative pion radiotherapy, physical and radiobiological aspects. In: Current topics in radiation research (M. Ebert et A. Howard, Eds. (North Holland publishing Co., 1974, 8, 159-233.

[11] WinAND L. Les particules élémentaires. Notes de cours à la Faculté des sciences appliquées, Université de Liège. Aspects de la physique moderne : les particules élémentaires. Bull. Soc. Roy. Belge des Électriciens, 1970, 4; 1971, 1 et 2.

[12] Yukawa H. Proc. Math. Soc. Japan, 1935, 17, 48. 